\title{
Percepção dos gestores do Sistema Único de Saúde acerca dos desafios da formação das Redes de Atenção à Saúde no Brasil
}

\author{
| ${ }^{1}$ Helena Eri Shimizu |
}

Resumo: O estudo teve como objetivo analisar as percepções de gestores sobre os desafios da formação de Redes de Atenção à Saúde no Brasil. Foram realizadas entrevistas semiestruturadas com sete gestores envolvidos com a formulação de políticas públicas de saúde. As falas foram analisadas com auxílio do software Analyse Lexicale par Contexte d'un Ensemble de Segments de Texte (ALCESTE). Identificaram-se três eixos temáticos. O primeiro foi denominado "A fragmentação do SUS dificulta o direito à saúde", e constituiu-se pela classe um, que trata dos fatores que contribuem para a fragmentação do sistema de saúde, e pela classe cinco, que aborda a saúde como direito social. O segundo eixo temático, denominado "O Estado na conformação das redes SUS”, é composto pelas classes dois, que demonstra as dificuldades na regionalização da saúde, três, que trata da governança regional, e quatro, que versa sobre a contratualização das redes regionais. O terceiro eixo, "Redes e a integralidade", é formado apenas pela classe seis, que aborda os desafios da formação de redes que garantam a integralidade da atenção. O estágio do desenvolvimento das redes regionais de atenção é considerado incipiente; todavia, a regulamentação da Lei Orgânica da Saúde, contendo diretrizes para a organização da regionalização, é considerada estratégica para organização das Redes de Atenção à Saúde.

> Palavras-chave: Redes de Atenção, Integralidade, Governança

\author{
1 Departamento de Saúde \\ Coletiva, Universidade de \\ Brasília. Brasília, Brasil. Endereço \\ eletrônico: shimizu@unb.br
}

Recebido em: 22/12/2012 Aprovado em: 04/12/2013 
No Brasil, as significativas mudanças demográficas que vêm sendo observadas apontam para o envelhecimento da população e um notável aumento das condições crônicas em seu perfil de morbimortalidade (BRASIL, 2011). Isso ocorre devido ao aumento da sobrevida de pacientes portadores de doenças crônicas ou de outras patologias, cujo controle tem sido viabilizado pelo desenvolvimento científico e tecnológico, que coexiste com as doenças infectocontagiosas e as mortalidades por causas externas, bastante expressivas.

Esse novo quadro epidemiológico, bastante complexo, exige mudanças na política de atuação do Sistema Único de Saúde (SUS), que se encontra fragmentado devido à fragilidade do processo de articulação entre as instâncias gestoras do sistema, entre os serviços e, ainda, entre as práticas clinicas desenvolvidas por diferentes profissionais, dificultando o acesso e a continuidade da atenção à saúde (LAVRAS, 2011). É, pois, necessária a adoção de um modelo que garanta a integração dos serviços de saúde, de modo a possibilitar o manejo clínico das condiçōes agudas e crônicas e garantir a continuidade assistencial (HARTZ; CONTANDRIOPOULOS, 2004; MENDES, 2011).

A estruturação das Redes de Atenção à Saúde apresenta-se como uma alternativa potente para reduzir a fragmentação do sistema, conforme demonstrado por alguns países que conseguiram consolidar sistemas de saúde integrados, que favoreçam o acesso, a continuidade, a integralidade e a racionalização dos recursos. Todavia, há que se considerar que as propostas de organização de redes têm diferentes conformações e significados e, portanto, exigem a análise da natureza dos sistemas, seus valores e princípios.

Esse modelo de organização de sistema de saúde foi descrito pela primeira vez em 1920 no Relatório Dawson (OPAS, 1964), que explicita formas de organizar um sistema de saúde regionalizado e hierarquizado, por nível de complexidade assistencial, tendo como base um território definido. As concepções desse modelo foram adotadas na criação do sistema nacional de saúde britânico, em 1948, o qual, por ter permitido acesso qualificado, sobretudo com baixo custo, 
tendo como porta de entrada a Atenção Primária à Saúde, passou a orientar a reorganização dos sistemas de saúde em vários países do mundo.

Países como a Espanha e o Canadá, que enfatizam o acesso universal e os princípios da regionalização, adotam o modelo de organização de redes como estratégia, com diferenças nos arranjos organizacionais, mas centrado no cuidado integral (KUSCHINIR; CHORNY, 2010). Mais recentemente, os Estados Unidos da América, que dispõem de um sistema de saúde bastante segmentado, com forte predominância do setor privado, introduziram um movimento de integração vertical, denominado integrated delivery systems (IDS), que se constitui de redes que proveem diversos tipos de serviços de saúde a uma população definida (MENDES, 2011; KUSCHINIR, CHORNY, 2010).

A Organização Panamericana de Saúde validou uma proposta de organização de redes integradas de serviços de saúde, baseada na Atenção Primária, devido à necessidade de delimitar os conceitos e modelos de redes para possibilitar a troca de experiências e a avaliação de resultados, definindo a rede como organização que provê ou faz arranjos para oferecer serviços de saúde equitativos e integrais a uma população definida e presta contas por seus resultados (OPAS, 2008).

No Brasil, a implementação de redes integradas de atenção do SUS, apesar de estar concebida constitucionalmente, pouco conseguiu avançar. Tal implementação fora destacada na Norma Operacional de Assistência de 2001 e, posteriormente, enfatizada com a publicação do Pacto pela Saúde, mais especificamente pelo Pacto de Gestão, que sublinha a cooperação solidária entre os municípios de uma determinada região (VIANA, 2011).

A política nacional de Atenção Básica $(\mathrm{AB})$, que propõe reorganizar o modelo de atenção a partir da Estratégia de Saúde da Família, foi um facilitador para a construção das Redes de Atenção à Saúde (CONILL, 2008; LAVRAS, 2011). $\mathrm{A} A \mathrm{~B}$ é tida como coordenadora das redes e do cuidado por meio das ações de promoção, prevenção e resolução dos agravos que possam demandar uma atenção de maior complexidade (SILVA, 2011). As ações de média complexidade são estruturadas nas unidades de referência secundárias, onde é possível realizar 
consultas, exames especializados e atendimentos de urgência e emergência de demanda espontânea ou de casos encaminhados pelas unidades de $\mathrm{AB}$.

Apesar das melhorias alcançadas com a expansão da $\mathrm{AB}$ no país e da indiscutível disseminação dos pressupostos que embasam essa estratégia, a fragmentação dos serviços é bastante presente, o que tem remetido à necessidade de buscar alternativas que favoreçam a organização mais sistêmica do sistema de saúde. Assim, o desafio de implementar sistemas regionais de saúde articulados em redes foi lançado oficialmente pelo Ministério da Saúde, através da Portaria no 4.279, de 30 de dezembro de 2010, que estabelece diretrizes para a organização da Rede de Atenção à Saúde no âmbito do Sistema Único de Saúde (BRASIL, 2010).

A publicação do Decreto no 7508, de 28 de junho de 2011, que regulamenta a Lei $n^{\circ} 8.080$, destaca novamente a discussão do aprimoramento das redes de atenção nas regiôes de saúde, e, portanto, coloca o desafio da construção das redes para além da perspectiva da organização dos serviços. Compreende-se por Redes de Atenção à Saúde, no SUS, uma malha que interconecta e integra estabelecimentos e serviços de saúde, organizados de forma a contemplar os diferentes níveis e densidades tecnológicas em um determinado território, visando ao atendimento integral aos usuários (SILVA JUNIOR, 2013).

Algumas redes temáticas têm sido construídas, como o Programa Mãe Curitibana, de atenção à mulher e à criança (JIMENEZ et al., 2001) e a iniciativa de Minas Gerais, em escala estadual, de estabelecer quatro redes temáticas: a rede de atenção à mulher e à criança (Rede Viva Vida), a rede de atenção às doenças cardiovasculares e diabetes (Rede Hiperdia), a rede de atenção aos idosos (Rede Mais Vida) e a rede de atenção às urgências e às emergências. Essas experiências têm apresentado resultados extremamente favoráveis e certamente contribuem para a conformação das Redes de Atenção à Saúde. Contudo, uma rede só consegue se concretizar se houver atores políticos comprometidos com sua efetividade (usuários, trabalhadores da saúde, gestores e toda a sociedade). Para isso, se faz necessária a criação de um sistema de governança adequado, onde a articulação e a cooperação entre os atores sociais e políticos e a construção de novos arranjos institucionais possam ocorrer (MENDES, 2011). 
A presente pesquisa teve como objetivo analisar a percepção de gestores do SUS envolvidos com a formulação de políticas de saúde sobre a formação de Redes Regionalizadas de Atenção à Saúde no Brasil.

\section{Metodologia}

Trata-se de estudo qualitativo que permite investigar as percepções, crenças e valores acerca de fenômenos sociais dinâmicos e complexos. Foram realizadas entrevistas semiestruturadas com sete gestores envolvidos com a formulação da política nacional de saúde, mais especificamente com a formulação da Rede de Atenção à Saúde. Foram entrevistados quatro secretários do Ministério da Saúde, dois ex-presidentes do Conselho Nacional de Secretários Municipais de Saúde e um diretor de desenvolvimento de Redes de Atenção do Ministério da Saúde. A escolha dos sujeitos da pesquisa se justifica devido a seu grande envolvimento com a formulação e gestão das políticas do SUS em diversos momentos históricos.

Para as entrevistas, foi empregado um roteiro semiestruturado, que abordou os aspectos relacionados ao processo de desenvolvimento das redes regionalizadas de atenção à saúde no Brasil. Todavia, foi estimulado que os sujeitos falassem livremente sobre o tema.

Para organização e análise dos dados, foram utilizados os recursos do software Analyse Lexicale par Contexte d'un Ensemble de Segments de Texte (ALCESTE), em sua versão 4.10, desenvolvido na França por M. Reinert. O software identifica co-ocorrências, ou seja, realiza análise lexical tendo a palavra como unidade, considerando-as nos seus respectivos contextos de ocorrência (REINERT, 1990), e possibilita conhecer a estrutura e a organização do discurso, fornecendo acesso aos universos lexicais. Em realidade, o programa auxilia na identificação do campo comum de ideias compartilhadas por um grupo social acerca de um determinado objeto social.

O programa realiza classificação hierárquica descendente, que permite análise lexicográfica do texto e oferece contextos (classes) caracterizados por seu vocabulário e pelos segmentos de texto compartilhados nesse vocabulário. Desta feita, o software permite, a partir de uma análise textual das produçôes 
semânticas dos sujeitos, utilizar a relação entre as palavras para identificar a frequência com que aparecem, bem como suas associações em classes (cálculo do $\chi 2$ ), e reconstituir o discurso compartilhado entre os sujeitos.

O projeto foi aprovado pelo Comitê de Ética em Pesquisa da Faculdade de Ciências da Saúde da Universidade de Brasília sob o número 123/11. Foi apresentado aos participantes um Termo de Consentimento Livre e Esclarecido, conforme recomenda a Resolução 196/96 do Ministério da Saúde.

\section{Resultados e discussão}

O ALCESTE identificou um corpus contendo sete Unidades de Contexto Inicial (UCI), divididas em 516 Unidades de Contexto Elementares (UCE), e classificou 378 UCE, o que representa 73,25\% do material submetido à análise.

A figura 1 demonstra que foram obtidas dos discursos seis classes, distribuídas em três eixos. O primeiro eixo, denominado "A fragmentação do SUS dificulta o direito à saúde", é constituído pela classe um, que contém $12 \%$ das palavras analisadas e problematiza os fatores que contribuem para a fragmentação do sistema, e pela classe cinco, que trata da saúde como um direito social, com 7\% das palavras analisadas. $\mathrm{O}$ segundo eixo, "O Estado na conformação das redes do SUS”, é formado pela classe dois, que aborda as dificuldades no processo de regionalização do SUS, contendo $16 \%$ das palavras, pela classe três, que discute a governança regional em saúde, com $11 \%$ das palavras analisadas, e pela classe quatro, contendo $7 \%$ das palavras, que discorre sobre a contratualização das redes nas regiōes de saúde. O terceiro eixo, "Redes e integralidade", é formado apenas pela classe seis, com $47 \%$ das palavras analisadas, e discorre sobre os desafios da conformaçãao das Redes de Atenção à Saúde no SUS. 


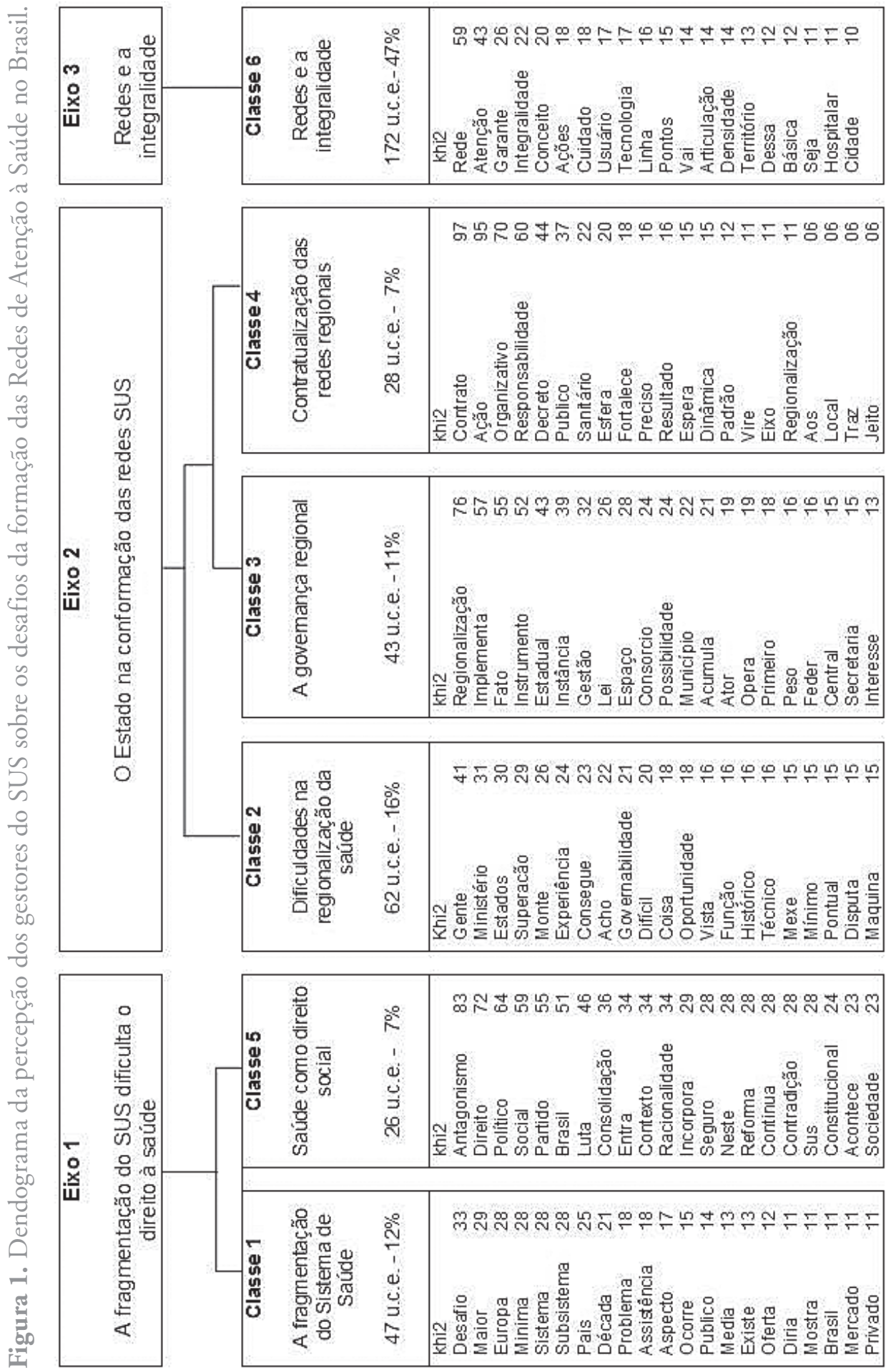




\section{Classe 1 - A fragmentação do sistema de saúde}

Nesta classe, os sujeitos tratam do desafio histórico de conformar a Rede de Atenção à Saúde, sobretudo para superar a fragmentação do sistema. Outro desafio apontado nesta classe é a necessidade de coordenação de serviços públicos e privados, que não ocorre no SUS porque o mercado age livremente por meio da oferta de serviços. É destacada também a necessidade de melhoria no financiamento e na formação profissional para o adequado funcionamento das RAS.

Foram destacadas pelos gestores as diferenças na organização dos sistemas de saúde entre os países, apontando os distintos contextos históricos na evolução das políticas de saúde. A luta contra a fragmentação do sistema de saúde consta como desafio na agenda dos países desde o século passado. Ademais, houve estágios de desenvolvimento distintos, quando se comparam o Brasil e os países da Europa e da América Latina (SILVA, 2011).

$\mathrm{Na}$ verdade, ela é uma agenda bem mais antiga. Se formos lembrar o início do século passado, na Inglaterra, quando o Relatório Dawson foi publicado, ele tinha como pressuposto essas ideias que nós estamos conversando. Como nós temos um problema estrutural maior que esses sistemas mais consolidados como os da Europa, evidentemente existe uma dificuldade maior; então nós estamos nessa situação intermediária entre os sistemas com maior dificuldade, como os latino-americanos, e outros com maior facilidade [...]. (Entrevistado 7)

A segmentação do sistema de saúde em subsistemas nacionais foi apontada pelos sujeitos como um dos fatores que influenciam a fragmentação, levando a diferentes estágios de integração nas diferentes regiōes do país.

Conforme afirmam Teixeira (2004) e Santos (2013), e também como ressaltam os gestores, concorre para a fragmentação do sistema a existência de dois projetos alternativos, em constante tensão: um hegemônico, o projeto neoliberal, e outro contra-hegemônico, representado pelo SUS, cujo modelo ainda não conseguiu se estabelecer. Por isso, ao conviver com forças políticas adversas instaladas no seio da sociedade brasileira, a manutenção do SUS é constantemente ameaçada.

Os gestores evidenciam que o mercado tem agido agressivamente com a oferta de serviços privados, sobretudo de média e alta complexidade, que se distribuem de forma bastante desigual no país, com o agravante de que não se conseguiu 
estabelecer mecanismos de cogestão entre o sistema público e esses serviços contratados, mantendo-se a modalidade de compra de serviços, o que acarreta baixa ou nenhuma integração às redes regionais. Esse quadro de frágil regulação do sistema induz à fragmentação que vem se agravando em virtude do recente processo de terceirização da gestão e da operação de grande parte da rede de serviços (organizações sociais, fundações privadas, parcerias público/privado).

A falta de investimento em equipamentos diagnósticos e terapêuticos e em tecnologia nos serviços públicos da Atenção Básica e nos serviços assistenciais de média complexidade levou a um crescimento desproporcional dos serviços privados complementares ao SUS (contratados e conveniados). Eles correspondem, hoje, a aproximadamente $65 \%$ das internações e a $92 \%$ dos serviços de diagnóstico e terapia (SANTOS, 2013).

Outro problema se refere à subvenção crescente do mercado dos planos privados de saúde com recursos federais, que ocorre por meio de: a) renúncia fiscal ou gastos tributários, b) cofinanciamento público de planos privados de saúde aos servidores federais do Executivo, Legislativo e Judiciário, incluindo as Estatais, e c) não ressarcimento obrigado pela Lei 9.656/1.998 (SANTOS, 2013).

Os gestores destacam, também, que contribui para a fragmentação do sistema a insuficiência de recursos financeiros para o SUS. Silva (2011) reforça que o financiamento atual é uma das principais dificuldades para que o sistema de saúde e, por conseguinte, as redes contem com recursos insuficientes para atender as necessidades assistenciais.

Tem a ver com a necessidade de condição mínima para estruturar os sistemas universais de saúde. Os sistemas universais de saúde para se consolidarem, precisam de um gasto público entre 6 a 8 por cento do PIB, mas nós estamos entre 3,4 por cento, então isso também é um desafio importante. (Entrevistado 7)

No documento do Projeto Mais Saúde, reconhece-se o subfinanciamento do SUS, se comparado tanto ao gasto per capita com outros países (6\% do Canadá, $11 \%$ da Espanha, 5,6\% do México,) quanto a indicadores simples, mas importantes, como o preço da consulta médica ou de uma cirurgia cardíaca (BRASIL, 2008). Nas suas diretrizes estratégicas e metas, o projeto recomendou o equacionamento desse problema por meio da regulamentação da legislação existente (Emenda Constitucional 29) e da participação adequada e estável da receita pública no financiamento da saúde, para assegurar à população brasileira a atenção universal, integral e equânime (BRASIL, 2008). 
Outro aspecto que, na percepção dos gestores, concorre para a fragmentação do sistema é a deficiente formação dos profissionais de saúde, que segue ainda orientação fortemente flexneriana, voltada para os interesses do mercado e, portanto, com muitas dificuldades para trabalhar em rede.

Como alerta Conill (2008), não é possível desconhecermos a necessidade de importantes mudanças culturais e no aparelho formador. Ou seja, urge a necessidade de se investir em formação profissional voltada para o alcance da integralidade da atenção. Essa lacuna fica mais evidente em comparação com outros países que conseguiram lograr avanços na integração de sistemas e de serviços, onde o médico generalista tem importância estratégica na ordenação e regulação do sistema a partir da Atenção Primária (MENDES, 2011).

\section{Classe 5 - Saúde como direito social}

Nesta classe, destaca-se a percepção dos sujeitos em relação à necessidade de continuar a luta da sociedade pela consolidação do direito à saúde no SUS como direito de cidadania. Os gestores reconhecem nesta classe que houve avanços, mas percebem que o SUS depara-se com diversas dificuldades para efetivação do direito à atenção integral à saúde. Além disso, reiteram que o SUS enfrenta políticas antagônicas, contrárias ao seu desenvolvimento, que têm levado à redução desse direito a oferta de serviços básicos.

Os gestores se colocam como protagonistas desse movimento, isto é, da luta da população pela consolidação do direito à saúde como condição de vida e bem estar social, o chamado Direito Social (DALLARI et al, 1996), que extrapola a visão de oferta de serviços e encontra ressonância com os direitos à dignidade humana (MACHADO, 2009).

Olhando para esse contexto, nós temos que entender que o nosso papel de defensores da ampliação dos direitos sociais, especialmente na área da Saúde, é fortalecer esse movimento. (Entrevistado 7)

Embora a constituição de 1988 aponte para a possibilidade de acesso às ações de saúde, no seu mais ampliado conceito, o cotidiano dos serviços mostra uma situação antagônica, na qual esse direito muitas vezes precisa ser reivindicado pela população. Segundo Dallari et al. (1996), quando o Estado se comporta dessa forma, deve ser constantemente pressionado pela sociedade civil, politicamente constituída, para que ela tenha seus direitos garantidos. Todavia, na percepção 
dos gestores, essa é uma fragilidade, porque a sociedade brasileira ainda não incorporou o SUS como um bem público a que tem direito.

Os gestores reiteram que o Estado brasileiro sofre grandes influências da racionalidade macroeconômica, que tem modificado a concepção de direito à saúde porque vem sendo concebida como um dos pilares do direito do consumidor. A associação da saúde ao consumo coloca em discussão a força do mercado, antagônica ao desenvolvimento do SUS. Essa nova forma de compreender o direito à saúde tem-se resumido a uma concepção de acesso aos bens e serviços de saúde. Santos' (2009) manifesta que nos últimos tempos ocorreu não só o arrefecimento das perspectivas e esperanças iniciais das camadas médias e dos servidores públicos em relação ao pertencimento ao SUS, como também um retrocesso na consciência social de direitos e pleitos, que se voltam para os planos privados de saúde por meio das entidades classistas, preferencialmente quando regulados e subsidiados pelo governo.

[...] a sociedade brasileira como um todo é que precisa incorporar o SUS constitucional nessa agenda de mudanças. (Entrevistado 7)

Para alguns autores, o SUS depara-se atualmente com um dilema: aprofundar a luta pela observância do SUS constitucional como conquista de direitos de cidadania (DRUCK; FILGUEIRAS, 2007; SANTOS, 2013) ou ceder às pressões impostas por um modelo de Estado que induz à crescente terceirização e a propostas de flexibilização das regras de controle na gestão de recursos humanos, dos serviços e dos recursos financeiros.

\section{Segundo Eixo - "O Estado na conformação das redes SUS"}

\section{Classe 2 - Dificuldades no processo de regionalização da saúde.}

$\mathrm{Na}$ classe dois, os discursos dos sujeitos voltam-se para o papel do Estado na estruturação da política sanitária visando à superação da fragmentação do sistema por meio do processo de regionalização. Os gestores falam, mais enfaticamente, da atuação do Ministério da Saúde na indução do processo de regionalização na saúde, bem como das dificuldades operacionais para implementar as redes regionalizadas.

Nesse sentido, os gestores recuperam as experiências que os Estados já acumularam historicamente na tentativa de superarem a fragmentação do sistema. Destacam que, dentre elas, a regionalização está na agenda estatal desde 
a publicação do Pacto pela Saúde, em 2006, que se propôs a substituir o formato rígido da Norma Operacional de Assistência à Saúde por uma pactuação mais flexível, que contribua para o processo de formação de redes regionais. Nesse momento, definiram-se como redes regionalizadas de atenção à saúde estruturas integradas de ações e serviços de saúde, institucionalizadas pela política pública em um determinado espaço regional a partir do trabalho coletivamente planejado e do aprofundamento das relações de interdependência entre os atores envolvidos.

Reconhece-se que houve alguns avanços, como a revisão dos marcos geográficos da regionalização, a constituição de colegiada gestora regional e a programação da oferta, mas foram mínimas as mudanças decorrentes da pactuação entre gestores para a integração regional (VIANA, 2011). Essas mudanças ocorreram, sobretudo, nos estados em que os Colegiados de Gestão Regional assumiram a agenda da regionalização (VIANA, 2011).

Para superar esses desafios, surge como alternativa a proposta de criação da rede interfederativa de saúde, por meio do Decreto $n^{\circ} 7.508$ e seus dispositivos (BRASIL, 2011), com o propósito de fortalecer os compromissos e responsabilidades entre os entes federados, permitindo avanços na institucionalidade do planejamento regional.

O ministério está muito mais próximo, porque pra gente conformar redes, isso implica numa mudança de processo de trabalho e o modo de fazer gestão, que significa em horizontalizar relações, se aproximar, porque o ministério teve historicamente uma função muito normativa, e os estados e municípios mais executores. (Entrevistado 2)

Nessa reflexão, os gestores apontaram a necessidade de o Estado adotar uma postura mais flexível, de diálogo com os entes federativos, que se inicia com a construção do planejamento integrado e regionalizado, e que deve priorizar as regiōes com maiores dificuldades para o desenvolvimento das redes do SUS, como o Norte e o Nordeste.

Oliveira (2008), ao analisar as desigualdades regionais na Amazônia, observa que há necessidade de estruturação e espacialização de mecanismos indutores. Estes seriam a interiorização do investimento na formação dos recursos humanos, através de cursos e de centros de formação e pesquisa. Outra condição apontada como essencial para a gestão eficaz dos serviços é a contribuição técnica e financeira dos Estados na implementação da política de regionalização, em novo processo de pactuação entre os entes. 
O mesmo autor, referindo-se à região amazônica, considera que é necessário

maior investimento financeiro nos seus serviços de saúde por parte do Governo Federal, além de uma política diferenciada que considere os desafios do processo de regionalização e a realidade epidemiológica do território (OLIVEIRA, 2008). Essa reflexão pode ser estendida também à região Nordeste.

Fleury e Ouverney (2007) apontam que para promover a regionalização é preciso rever quem custeia as ações e procedimentos que compóem as referências intermunicipais nas regiōes, como também é necessário realizar investimentos para ampliar o acesso universal, equitativo e integral. Nesta classe, os sujeitos apontam as dificuldades de gestão do processo de regionalização em saúde no país, sobretudo devido à fragilidade da capacidade técnica dos entes envolvidos o Ministério da Saúde, os estados e municípios -, que não estão adequadamente preparados para conduzir o processo de gestão compartilhada.

Temos um plano que é bom, temos governabilidade para operacionalizar, temos uma limitação que é a capacidade de gestão... do ministério, mas eu acho que a gente pode ampliar que aqui acaba refletindo em estados e municípios. (Entrevistado 2)

Fica também evidente nesta classe que os gestores percebem que, além da dificuldade técnica, outro problema a ser superado é a dimensão política, que envolve disputas e conflitos que devem ser enfrentados para implantação do processo de regionalização.

Eu acho que tem um entendimento muito harmônico dos grandes eixos; não temos dificuldades conceituais, temos dificuldades operacionais de governança, de sustentabilidade que, às vezes, têm contradições no campo da política. (Entrevistado 3)

O território regional deve permitir ao cidadão satisfazer as suas necessidades de saúde, por meio da oferta adequada de serviços, permitindo superar as desigualdades sociais, emanadas em grande parte das desigualdades territoriais (SANTOS, 2011).

\section{Classe 3 - A governança regional}

Nesta classe, os gestores expressam a preocupação com a governança das redes regionais de saúde a ser construída entre os entes federativos, pois se trata de uma experiência nova de cogestão, na qual haverá mudanças nas formas como cada ator operará no contexto das regiōes de saúde. 
Nesse sentido, os gestores revelam grande preocupação com a conformação da governança regional, pois as Secretarias Estaduais de Saúde quase não têm instrumentos de cogestão com os municípios. Ademais, ressalta-se que a região de saúde requer estrutura de governança que só poderá ser empregada para justificar mudanças estruturais visando alterar a distribuição do poder em contextos específicos:

Pra mim é a grande fragilidade: qual a instância de governança que vai fazer isso? Ela [a região] tem instrumentos e arranjos de gestão pra dar conta disso? Tem um espaço virtual de pactuação de compromissos, mas não é uma instância de gestão regional, não tem instrumentos, não está empoderada, não tem esse poder. (Entrevistado 1)

De acordo com Santos e Andrade (2011),

a rede interfederativa é a interconexão dos entes políticos responsáveis pela saúde, que devem, conjuntamente, em espaços como colegiados, decidir, de comum acordo, todas as questôes referentes à prestação de serviços em rede, padrão de integralidade, critérios de incorporação tecnológica, regulamentos técnicos, protocolos clínicos, fiscalização, controle, avaliação e outros aspectos do sistema.

O Decreto permite você repassar recursos com base regional [...], abre uma possibilidade, e a Lei $n^{\circ} 141$, que regula a Lei $n^{\circ} 29$, permite que um ente repasse recurso para outro. É possível o fundo municipal e o fundo estadual ter o repasse de recursos sem a necessidade de convênios. (Entrevistado 1)

A governança das redes no SUS, portanto, dependerá da capacidade de articulação e pactuação na Comissão Intergestores Regional e de uma boa construção do Contrato Organizativo de Ação Pública, previsto no Decreto 7508/2011 (BRASIL, 2011). Esse contrato deve ser elaborado de modo participativo, com a definição das responsabilidades, das metas, da organização, do financiamento e da integração das açōes e serviços de saúde, sob a responsabilidade dos entes federativos na região.

O processo de governança das regiōes de saúde dependerá de como ela é concebida, de quem a integra, dos propósitos da sua formação, do contexto em que ela é formada, da sua extensão, dos recursos disponíveis para serem integrados ou trocados, dos mecanismos de coordenação empregados e das açōes de outros atores diante de sua formação. Esse aspecto foi reforçado pelos gestores, que avaliam como incipiente o estágio atual do desenvolvimento das redes regionais de saúde e apontam a existência de diversos desafios para a construção da governança compartilhada. 


\section{Classe 4 - Contratualização das redes regionais}

Nesta classe, a percepção dos gestores remete à necessidade de se contar com um instrumento jurídico na definição das responsabilidades dos entes federativos na constituição das redes regionais.

Os COAPs [Contrato Organizativo de Ação Pública], onde as esferas Federal, Estadual e Municipal teriam definidas neste contrato suas responsabilidades, parte do pressuposto de que a gestão intergovernamental precisa ter uma institucionalidade jurídico-legal mais adequada? Vai funcionar? Tenho dúvidas, eu acho que poderá representar um avanço, mas qual vai ser a dificuldade? (Entrevistado 7)

Foi relatada pelos gestores a preocupação com a indefinição de papéis em relação a qual a esfera de governo que assumirá a responsabilidade de assegurar o acesso a serviços de maior densidade tecnológica, inexistentes naquela região. Do mesmo modo, há receio de que haverá dificuldades em garantir que alguns estados contribuam com o financiamento adequado para as regiões de saúde, como tem ocorrido historicamente no país. Nesse sentido, verificou-se a percepção de alguns gestores de que o Decreto 7508/2011, ao instituir o contrato organizativo de ação pública, conferirá mais força aos pactos e, portanto, poderá auxiliar na correção de iniquidades, por meio da responsabilização dos atores e entes envolvidos na articulação regionalizada das redes de cuidados integrais.

Santos e Andrade (2013) esclarecem que o contrato de ação pública é um contrato administrativo plurilateral que se configura como um acordo de vontades, do qual participam pessoas administrativas, obrigadas reciprocamente a promover condutas visando à obtenção de resultados de interesse coletivo. Em recente artigo, Andrade (2012) avalia que o contrato traz maior segurança jurídica aos entes participantes da região de saúde, sem o que, até então, se mantinha frágil a regionalização. É fundamental observar que os contratos deverão ser construídos e celebrados entre os entes da região de saúde, com definição e acompanhamento pelas respectivas Comissões Intergestores Regionais (SANTOS; ANDRADE, 2011).

A administração pública por contrato tem sido empregada em vários países, como França, Itália e Espanha, logrando sucesso na oferta de serviços de saúde necessários à população que vive em um território (LÓPEZVALCÁRCEL, 2006). 
Terceiro Eixo - Redes e a integralidade

Classe 6 - Redes e a integralidade

Emerge nesta classe a percepção dos gestores sobre a necessidade de relação entre as redes regionalizadas e integradas de atenção e a integralidade do cuidado em saúde. As redes são percebidas como arranjos organizativos que possibilitam a continuidade do cuidado e o alcance do princípio constitucional da integralidade. São descritas pelos gestores como um conjunto de ações e serviços de saúde de diferentes densidades tecnológicas, que devem se articular para ofertar diferentes tipos de serviços:

Eu faço uma divisão de redes assistenciais e redes de serviço onde eu acho que a Atenção Primária é uma rede importante. A atenção especializada é outra rede que muitas vezes está implicada com a rede hospitalar. A urgência e emergência acabam sendo outra rede e a saúde mental também se configura como uma rede importante. Eu sempre trabalho com a ideia de 5 grandes redes que se articulam. (Entrevistado 5)

Aqui, o conceito de integralidade está ligado, portanto, ao de integração de serviços por meio de redes assistenciais, que reconhece a interdependência dos atores e das organizaçóes, tendo em vista que nenhuma delas dispóe da totalidade dos recursos e competências necessários para a solução dos problemas de saúde de uma população em seus diversos ciclos de vida (HARTZ; CONTANDRIOPOULOS, 2004).

A integração entre os participantes da rede foi também considerada pelos gestores condição essencial, posto ser fundamental estabelecer mecanismos de cooperação e coordenação adequados e comprometidos com os recursos coletivos para responder às necessidades de saúde em âmbito regional. Essa forma de organização, que Hartz e Contandriopoulos (2004) consideram imagem ideal e denominaram um "sistema sem muros", se daria com a eliminação das barreiras de acesso entre os diversos níveis de atenção - do domicílio até o hospital, generalistas, especialistas, setores público e privado - unidos por corredores virtuais que conduzem para além dos limites municipais, otimizam recursos e ampliam oportunidades de cuidados. Nesse sentido, tem-se afirmado que a integralidade da atenção é um eixo prioritário da investigação e avaliação dos serviços e dos sistemas de atenção à saúde, estruturados como redes assistenciais interorganizacionais que articulam as dimensões clínicas, funcionais, normativas e sistêmicas para a solução dos problemas de saúde de uma população, em seus diversos ciclos de vida (HARTZ; CONTANDRIOPOULOS, 2004). 
Para os gestores, as redes temáticas são linhas de cuidado, e por si só não constituem Redes de Atenção à Saúde, mas podem contribuir para a sua construção. Segundo eles, atualmente, organizam-se redes temáticas no país que servem para facilitar o diálogo e orientar os usuários sobre o itinerário que devem percorrer nas linhas e pontos da rede.

As redes temáticas apresentam potencial para avançar na perspectiva da integralidade vertical, proposta por Magalhães e Mattos (2006), a qual implica a busca da satisfação da necessidade dos usuários por meio de práticas que permitam cuidados integrais.

Para termos as redes de cuidados integrais, é preciso qualificar a Atenção Primária para exercer sua atribuição de coordenação do cuidado e também organizar pontos de atenção especializada integrados, intercomunicantes, que forneçam aos usuários do SUS resposta adequada, levando em consideração o diagnóstico das necessidades em saúde aplicado à diversidade e à extensão do território brasileiro (MENDES, 2007; 2011).

Para os gestores, o melhor desenho de rede é aquele que responde às necessidades de saúde da população de determinado território, o que implica, por um lado, usar a criatividade, não propondo mudanças impossíveis de ser realizadas, e, por outro, não se prender a parâmetros tradicionais de oferta e organização de serviços, buscando inovar o modelo assistencial (SILVA, 2008). $\mathrm{O}$ desenho das Redes de Atenção à Saúde deve ser realizado por meio de uma relação dialética dos princípios de economia de escala, de escopo e de qualidade no acesso aos serviços.

Mendes (2011) reafirma não ser conveniente o emprego de modelos rígidos para a implantação de redes, devido a diferentes realidades econômicas, culturais e sanitárias vigentes no Brasil. Para ele, o mais adequado é a adoção de diretrizes operacionais, as quais podem ser flexibilizadas para se adaptarem às diferentes realidades.

Nesta classe, reitera-se que, para o bom funcionamento das redes, prescindese do fortalecimento da governança regional; há noção do imenso desafio de se construírem regiōes de saúde integrando entes autônomos em um processo de interdependência e cooperação interfederativa (FLEURY; OUVERNEY, 2007).

Em suma, as Redes de Atenção à Saúde devem permitir o resgate da integralidade, que é um dos pilares do SUS, sua imagem-objetivo, e que tem como 
fundamentos a garantia de atenção nos três níveis de complexidade assistencial, a articulação das açōes de prevenção, promoção e recuperação e a abordagem integral dos indivíduos e das famílias (GIOVANELLA et al., 2002).

\section{Considerações finais}

A conformação das Redes de Atenção à Saúde no SUS ainda é um grande desafio, que requer forte investimento do Estado para a sua consolidação. Para tanto, o Estado deve ter uma política que impulsione e apoie as redes como estratégia primordial para o alcance de serviços de saúde de qualidade para toda a população. Nessa perspectiva, a constituição das redes é entendida como importante instrumento de garantia do direito à saúde por permitir ampliar o acesso e reduzir as desigualdades regionais, que ainda são imensas em nosso país.

O processo de implantação do Decreto no 7508 e de seus dispositivos é ainda recente e, portanto, não permite avaliações mais profundas. Todavia, a operacionalização desse processo não pode seguir modelos prescritivos, e requer investimentos que incluam o aprimoramento das dimensões técnicas e políticas. Da dimensão política, há que se considerar que ocorrem disputas de poder e conflitos no âmbito regional, inclusive as formatadas pelo sistema de serviços de saúde privado, mas que requerem fortes mecanismos de regulação, pois devem convergir para preservar a responsabilidade sobre as condições de saúde e de acesso da população às ações e serviços. Nessa perspectiva, a capacidade técnica diz respeito às estratégias de contratualização para a garantia da provisão de açôes e serviços de saúde de diferentes densidades tecnológicas, bem como à logística para orientar o usuário no itinerário das malhas da rede, sistema de regulação, com normas e protocolos claros para orientar o acesso à rede de serviços, definir competências e responsabilidades.

Finalmente, há que se ressaltar a importância desses processos serem alicerçados na valorização e no reconhecimento da Atenção Básica como instância responsável pelo ordenamento do sistema e pela coordenação do cuidado. É preciso resgatar seu papel, constituí-la como a principal porta de entrada do sistema, organizar o processo de trabalho de equipes multiprofissionais na perspectiva de abordagem integral do processo saúde-doença e garantir o acesso de cada usuário a qualquer outra unidade funcional do sistema em função das suas necessidades. ${ }^{1}$ 


\section{Referências}

ANDRADE, L.O.M. Inteligência de governança para apoio à tomada de decisão. Ciência \& Saúde Coletiva, v. 17, n.4, p. 829-832, 2012.

BRASIL. Ministério da Saúde. Portaria GM/MS no 4.279, de 30 de dezembro de 2010. Estabelece diretrizes para a organização da Rede de Atenção à Saúde no âmbito do Sistema Único de Saúde (SUS). Diário Oficial da Uniāo, Poder Executivo, Brasília, DF, 31 dez 2010. Seção 1.

. Ministério da Saúde. Redes regionalizadas de atenção à saúde: contexto, premissas, diretrizes gerais, agenda tripartite para discussão e proposta de metodologia para implementação: versão para debate. Brasília: MS, 2008.

- Ministério da Saúde. Secretaria de Gestão Estratégica e Participativa. Decreto no 7.508, de 28 de junho de 2011. Regulamenta a Lei no 8.080, de 19 de setembro de 1990, para dispor sobre a organização do Sistema Único de Saúde - SUS, o planejamento da saúde, a assistência à saúde e a articulação interfederativa, e dá outras providências. Diário Oficial da União, Poder Executivo, Brasília, DF, 29 jun. 2011. Seção 1, p.1-3.

CONILL, E.M. Ensaio histórico-conceitual sobre a Atenção Primária à Saúde: desafios para a organização de serviços básicos e da Estratégia Saúde da Família em centros urbanos no Brasil. Cad. Saúde Pública, v. 24, supl. 1, p. s7-s16, 2008.

DALLARI, S.G. et al. O direito à saúde na visão de um conselho municipal de saúde. Cadernos de Saúde Pública, Rio de Janeiro, v. 12, n. 4, p. 531-540, 1996.

DRUCK, G.; FILGUEIRAS, L. Política social focalizada e ajuste fiscal: as duas faces do governo Lula. Rev Katál, v. 10, n. 1, p. 24-34, 2007.

FLEURY, S.; OUVERNEY, A.M. Gestão de redes: a estratégia de regionalização da política de saúde. Rio de Janeiro: FGV; 2007. 204p.

GIOVANELLA, L. et al. Sistemas Municipais de Saúde e a diretriz da integralidade da atenção: critérios para avaliação. Saúde em Debate, v. 26, n. 60, p. 37-61, 2002.

HARTZ, Z.M.A.; CONTANDRIOPOULOS, A.P. Integralidade da atenção e integração de serviços de saúde: desafios para avaliar a implantação de um "sistema sem muros". Cad. Saúde Pública, v. 20, supl. 2, p.331-336, 2004.

JIMENEZ, E.J.B. et al. Avançando na atenção materno-infantil: Programa "Mãe Curitibana”. In: DUCCI, L; Simão, M.B.G; Moisés, S.G. (Org.). Curitiba: a saúde de braços abertos. Rio de Janeiro: CEBES, p.201-212, 2001.

KUSCHNIR, R.; CHORNY, A.H Redes de atenção à saúde: contextualizando o debate. Ciência \& Saúde Coletiva, v. 15, n. 5, p. 2307-2316, ago 2010.

LAVRAS, C. Atenção primária à saúde e a organização de redes regionais de atenção à saúde no Brasil. Saúde e Sociedade, v. 20, n. 4, p. 867-874, 2011. 
LÓPEZ-VALCÁRCEL, B.G. Políticas contractuales en atención especializada. In: REPULLO, J.R.; IÑESTA, A. (Orgs.). Sistemas y servicios sanitarios. Madri: Diaz de Santos, 2006.

MACHADO, F.R.S. O direito à saúde na interface entre sociedade civil e Estado. Trab. educ. saúde, v. 7, n. 2, p. 355-371, 2009. 1.

MATTOS, R. A. Os sentidos da integralidade: algumas reflexões acerca de valores que merecem ser defendidos. In: PINHEIRO, R.; MATTOS, R. A. (Org.). Os sentidos da integralidade na atenção e no cuidado à saúde. Rio de Janeiro: Cepesc, 2009. p. 43-68.

MENDES, E.V. A modelagem das redes de atenção à saúde. Belo Horizonte: Secretaria de Estado da Saúde de Minas Gerais; 2007.

. As redes de atenção à saúde. Brasília: OPAS, 2011.

MINAS GERAIS. Secretaria de Estado de Saúde. Nota técnica no 3 do Comitê de Assuntos Estratégicos da SESMG: As prioridades do Plano de Governo para o periodo 2003/2006. Belo Horizonte, 2004.

OLIVEIRA, P.T.R. Desigualdade regional e o território da saúde na Amazônia. Belém: EDUFPA, 2008.

ORGANIZAÇÃO MUNDIAL DA SAÚDE. Relatório Mundial da Saúde 2008: Cuidados de Saúde Primários: agora mais que nunca. Genebra: OMS, 2008.

ORGANIZACIÓN PANAMERICANA DE LA SALUD. Informe Dawson sobre al futuro de los servicios médicos y afines, 1920. Washington, D.C.: OPAS, 1964. 38p. (OPS Publicación Científica, 93).

REINERT, M. Alceste une méthodologie d'analyse des données textuelles et une application: Aurelia De Gerard De Nerval. Bull Methodol Sociol. 1990; 26 p. 24-54.

SANTOS, L; ANDRADE, L.O.M. SUS o espaço da gestão inovada e dos consensos interfederativos. Campinas: IDISA, 2007.

Redes interfederativas de saúde: um desafio para o SUS nos seus vinte anos. Ciência \& Saúde Coletiva, v. 16, n. 3, p. 1671-1680, 2011.

SANTOS, N.R. A Reforma Sanitária e o Sistema Único de Saúde: tendências e desafios após 20 anos. Saúde em Debate, v. 33, n. 81, p. 13-26, 2009.

. SUS, política pública de Estado: seu desenvolvimento instituído e instituinte e a busca de saídas. Ciência \& Saúde Coletiva, v. 18, n. 1, p. 273-280, 2013.

SILVA, F. S. Organização de redes regionalizadas e integradas de atenção à saúde: desafios do Sistema Único de Saúde (Brasil). Ciência \& Saúde Coletiva. Rio de Janeiro, v. 16, n. 6, p.2753-2762, 2011.

SILVA, S.F. et al. Redes de atenção à saúde no SUS: o pacto pela saúde e redes regionalizadas de ações e serviços de saúde. São Paulo: Idisa, 2008. 
TEIXEIRA, S.A. Avaliação dos usuários sobre o programa de saúde da família em Vitória da Conquista, Bahia, Brasil. In: FERNANDES, A.A; SECLEN-PALACIN, J.A. (Org.). Experiências e desafios da atenção básica e saúde familiar: caso Brasil. Brasília: OPAS, 2004. VÁSQUEZ NAVARRETE, M.L.; VARGAS LORENZO, I. Organizaciones sanitárias integradas: un estúdio de casos. Barcelona: Consorci Hospitalari de Catalunya, 2007.

VIANA, A.I.D.; LIMA, L.D. O processo de regionalização na saúde: contextos, condicionantes e papel das Comissões Intergestoras Bipartites. In: . Regionalização e relaçôes federativas na politica de saúde do Brasil. Rio de Janeiro: Contra Capa, 2011.

\section{Nota}

${ }^{1}$ Agradecemos a Joseney Santos, que coletou os dados para a dissertação de mestrado defendida no Programa de Pós-Graduação em Ciências da Saúde. 


\section{Perception of managers of the Unified Health System about the challenges in the formation of Health Care Networks in Brazil}

The study aimed to analyze the perceptions of managers about the challenges in the formation of Health Care Networks in Brazil. Semistructured interviews with seven managers involved with the formulation of public health policies were carried out. The speeches were analyzed with the aid of software Analyse Lexicale par Contexte d'un Ensemble de Segments de Texte (ALCESTE). We identified three main themes. The first was called "Fragmentation of SUS hinders the right to health", and was constituted by class one, which addresses factors that contribute to the fragmentation of the health system, and class five, which addresses health as a social right. The second main theme, called "The State of SUS in shaping networks", is composed of class two, which shows the difficulties in the regionalization of health; three, which deals with regional governance; and four, which deals with the contracting of regional networks. The third axis, "Networks and completeness", is formed only by class six, which addresses the challenges of networking to ensure comprehensive care. The stage of development of regional networks of care is considered incipient, yet the regulation of Health Law containing guidelines for the organization of regionalization is considered strategic for the organization of Health Care Networks.

> Key words: Regionalized Heal thcare Networks; regionalization; Unified Health Care System. 